\title{
A Component Analysis of the Mindfulness-Based Positive Behavior Support (MBPBS) Program for Mindful Parenting by Mothers of Children with Autism Spectrum Disorder
}

\author{
Nirbhay N. Singh ${ }^{1} \cdot$ Giulio E. Lancioni ${ }^{2} \cdot$ Oleg N. Medvedev $^{3} \cdot$ Yoon-Suk Hwang $^{4} \cdot$ Rachel E. Myers $^{5}$ \\ Published online: 11 May 2020 \\ (C) Springer Science+Business Media, LLC, part of Springer Nature 2020
}

\begin{abstract}
Objectives Mindfulness-Based Positive Behavior Support (MBPBS) has been shown to be effective in reducing stress and burnout in parents and professional caregivers of children and adolescents with intellectual and developmental disabilities (IDD) and autism spectrum disorder (ASD). The aim of this study was to assess the comparative effects of the mindfulness (MB) and positive behavior support (PBS) components against the MBPBS program for mindful parenting.

Methods The study utilized a three-arm randomized controlled trial design, with a 10-week pre-treatment control condition, 30 weeks of intervention, and 3 years of post-intervention follow-up. Mothers of children with ASD were randomly assigned to the MB, PBS, and MBPBS conditions and provided 3 days of training specific to each condition. The effects of these programs were assessed on the mothers (i.e., training attendance, meditation time, perceived psychological stress) and spillover effects were assessed on their children with ASD (i.e., aggression, disruptive behavior, compliance with mothers' requests).

Results Mothers in the MBPBS condition reported greater reductions in perceived psychological stress, followed by those in the MB condition, and with no significant changes reported by those in the PBS condition. Reduction in the children's aggression and disruptive behavior followed a similar pattern, with most to least significant reductions being in MBPBS, MB, and PBS condition, respectively. Significant increases in compliance (i.e., responsiveness to mothers' requests) were largest in the MBPBS condition, followed by MB, and then PBS. Changes across all variables for both mothers and their children were maintained for 3 years post-intervention. After time and training type were controlled for, meditation time was a significant predictor in reducing aggressive and disruptive behaviors, and in enhancing compliance of the children with mothers' requests. Conclusions Positive outcomes for mothers and their children with ASD were significantly greater in the MBPBS condition, followed by the MB condition, and least in the PBS condition. MBPBS appears to be an effective mindful parenting program on the assessed variables.
\end{abstract}

Keywords MBPBS - Mindfulness - Perceived stress · Aggressive behaviors - Disruptive behaviors $\cdot$ Challenging behaviors Compliance

Many parents report that having a child is a watershed event in their lives, which brings much joy and happiness. For others, parenthood can be a source of stress and burnout, especially when the child begins to display emotional problems and
Nirbhay N. Singh

nirbz52@gmail.com

1 Department of Psychiatry and Health Behavior, Medical College of Georgia, Augusta University, Augusta, GA 30912, USA

2 Department of Neuroscience and Sense Organs, University of Bari, Bari, Italy
School of Psychology, University of Waikato, Hamilton, New Zealand

4 Institute for Learning Sciences and Teacher Education, Australian Catholic University, Brisbane, Queensland, Australia

5 WellStar School of Nursing, Kennesaw State University, Kennesaw, GA, USA 
behavioral challenges (Poole 2003; Roskam et al. 2017). In the general population, research suggests a strong bidirectional correlation between child psychopathology and psychological distress in their parents beyond what can be accounted for by shared genetic and environmental effects (Bagner et al. 2013; Stone et al. 2016; Yorke et al. 2018). Generally, parents who have a child with disabilities report high levels of stress, with parents of children with autism spectrum disorder (ASD) reporting the highest levels when compared with parents of children with intellectual disabilities and physical or other disabilities (Hayes and Watson 2013). While this is generally true, some parents also report many positive aspects of parenting children with ASD that have enhanced their own quality of life (Hastings et al. 2005; ten Hoopen et al. 2020). In terms of helping these parents, the issue is how to reduce parental stress and enhance the positive aspects of parenting children with ASD.

In the field of developmental disabilities, the general approach taken has been to provide behavioral parent training and psychoeducation programs that focus on helping parents to reduce their children's behavioral excesses and overcome their behavioral deficits (McIntyre and Neece 2016), but not on the psychological needs of the parents (Yu et al. 2019). Examples of evidence-based programs include Parent-Child Interaction Therapy (e.g., Bagner and Eyberg 2007; Bagner et al. 2010), Incredible Years Parent Training (Kleve et al. 2011; McIntyre (2008a, 2008b), and Stepping Stones Triple P program (Plant and Sanders 2007; Whittingham et al. 2009). Of these approaches, research suggests that Parent-Child Interaction Therapy is effective in reducing both parental stress and child maladaptive behavior (McIntyre and Neece 2016). In addition to behavioral parent training, mindfulness-based programs have emerged as another evidence-based approach for relieving stress in parents of children with intellectual and developmental disabilities (IDD) and ASD (Hwang and Singh 2016). For example, Neece (2014) reported that MindfulnessBased Stress Reduction reduced parental stress but had no effect on the children's internalizing or externalizing behaviors. However, other studies using customized mindfulness-based programs have shown positive effects in reducing parental stress, decreasing child behavior problems, and increasing child social interactions (e.g., Singh et al. 2006, 2007).

Mindfulness-Based Positive Behavior Support (MBPBS) is an example of a customized mindfulness-based program for mindful parenting (Singh et al. 2020a). MBPBS consists of two components - mindfulness-based practices (MB) and positive behavior support (PBS). It is the seamless braiding of two evidence-based components used by parents for personal transformation through formal and informal mindfulness practices and instructions in PBS which enables them to learn effective ways of positively managing the behavior of their children. An indirect effect of practicing mindfulness in daily life is the reduction of parents' psychological stress and anxiety, compassion fatigue, and burnout. The direct effect of using PBS is the reduction in the frequency, duration, intensity, and severity of challenging behaviors in their children. The focus of MBPBS is not on behavior change per se but on reducing the suffering of parents and their children through disciplined practice of meditation and the mindful use of PBS in interactions with their children.

In an early study, Singh et al. (2006) evaluated the cascading effects (Fowler and Christakis 2010) of parents engaging in a customized mindful parenting program (a precursor of the MBPBS program) on their children's aggression, non-compliance, and self-injurious behavior. Results indicated not only an increase in the parents' satisfaction with their parenting skills and interactions with their children with ASD but also decreases in the children's challenging behaviors (i.e., physical aggression, non-compliance, and self-injury). Singh et al. (2007) replicated and extended these findings with parents of children with IDD. The results included parents' increased satisfaction with their parenting skills, more positive social interaction with their children, and decreased parenting stress. In addition, spillover effects included decreased aggressive behavior and increased social behavior in their children. In the third study, with parents of children with attention deficit hyperactivity disorder (ADHD), Singh et al. (2010) evaluated the effects of mindful parenting by first teaching mindfulness practices to the parents alone and then providing similar training to their children with ADHD. The results for the parents were similar to those reported in previous studies, but the changes in the children's behavior were enhanced when they also received training in mindfulness practices.

In a proof-of-concept study, mothers of adolescents with ASD were taught an 8-week MBPBS program and the effects of the program were assessed on the mothers and on their adolescent children (Singh et al. 2014). No training was provided to the adolescents. Results showed that the adolescents' physical aggression and incidents of disruptive behavior significantly decreased, and compliance with mothers' requests significantly increased following the mothers' training in MBPBS. In addition, the mothers reported significant decreases in their psychological stress. In a two-arm study, mothers of adolescents with IDD were stratified into either an ASD group or an IDD alone group (i. e., with no ASD), with both groups of mothers receiving the 3-day MBPBS training program (Singh et al. 2019). The aim of the research was to test the suggestion currently prevalent in the research literature that, given the much higher prevalence of parental stress in parents of children with ASD, interventions need to be customized for the specific challenges faced by these parents (Cachia et al. 2016). Results showed no differential effects of MBPBS training on the stress level of both groups of mothers, or in the adolescents' aggressive, disruptive behavior, and compliance behaviors. This study suggested that training in MBPBS is equally effective with mothers of 
adolescents with ASD or IDD alone regardless of the pretraining level of their psychological stress.

Extant research on MBPBS suggests that this multicomponent program is effective in assisting parents to reduce their psychological stress, increase their satisfaction with their parenting, and enhance parent-child social interactions. In addition, the spillover or cascading effects of parent mindfulness change the behavior of their children regardless of whether the children have ASD or IDD alone. An initial study of the component effects of MBPBS with professional caregivers assessed the effects of the PBS intervention vs. MBPBS program (Singh et al. 2020b). While both the MBPBS program and the PBS intervention alone were effective, the MBPBS program produced significantly better outcomes for the caregivers, individuals with IDD, and the service provider agency. The aim of the present study was to extend the component analysis research on MBPBS and test the individual effects of MB vs. PBS vs. MBPBS, such that the specific effects of the two major components alone could be tested against their combined effects. In addition, this study extended the component analysis research to parents of children with ASD. Specifically, we tested the following hypotheses: (1) positive changes in parental behaviors will be highest in the MBPBS condition, followed by the MB alone condition, and then the PBS alone condition; and (2) the cascading effects of parental mindfulness and knowledge of how to mindfully intervene behaviorally with their adolescent's challenging behaviors will be greatest in the MBPBS condition, followed by the $\mathrm{MB}$ alone condition, and then the PBS alone condition.

\section{Method}

\section{Participants}

The participants were mothers of children with ASD. The mothers were recruited through various sources, including
Fig. 1 Participant flow through the trial

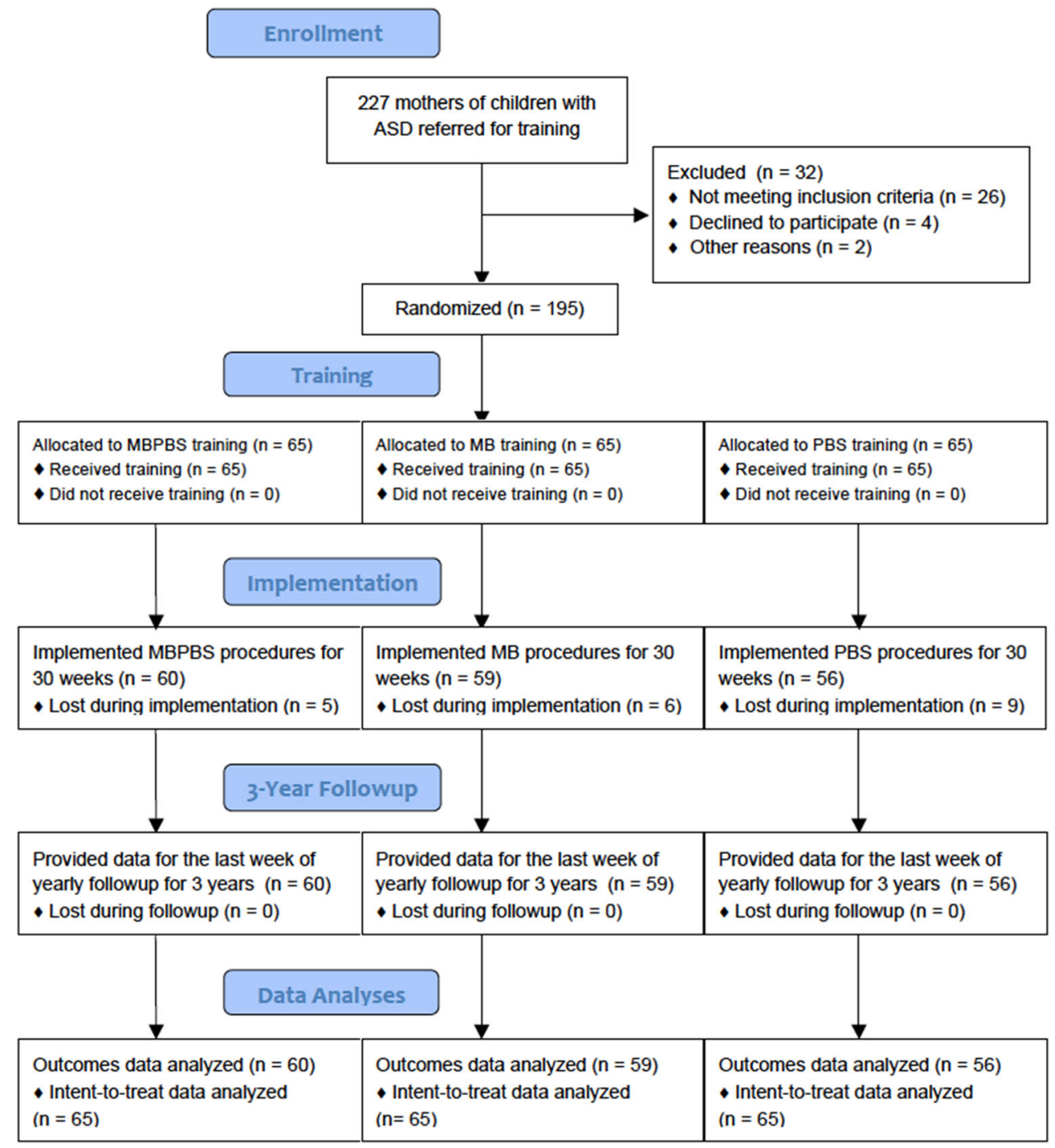


disabilities parent groups, listserv groups, self-referrals, flyers posted at family physician offices, pediatrician, and child psychiatry offices and clinics, and parent-toparent referrals. This recruiting resulted in an initial sample of 227 mothers of children with ASD. The nature of the research, study design, confidentiality issues, and their choice to enroll or not, as well as the ability to opt out of study at any time, were explained to the mothers. From the initial sample, 32 were excluded because of failure to meet the inclusion criteria (chiefly that the children have a firm diagnosis of ASD), declined to participate due to time and other family constraints, and for personal reasons. Using a random number generator, the remaining 195 mothers were randomized into the MBPBS, MB, or PBS experimental conditions. A CONSORT participant flowchart is presented in Fig. 1. Sociodemographic characteristics of the mothers and their children are presented in Table 1.

\section{Procedure}

\section{Experimental Design}

This was a three-arm randomized controlled trial (RCT), with a 10-week pre-intervention control condition. The experimental condition included a 3-day training in each active experimental condition (i.e., MBPBS, MB, and PBS). The training in each experimental condition was followed by 30 weeks of implementation. Finally, there was a follow-up condition during which the outcome variables were monitored each year for 3 years.

\section{Control Condition}

During the 10-week pre-intervention control condition, no experimental variables were introduced, and the mothers continued their usual parenting practices.

\section{Experimental Conditions}

MBPBS Condition In this condition, the 3-day stepped care version of the MBPBS protocol was used for teaching mindfulness, related meditation practices, and guiding principles of positive behavior support to the mothers (see Table 2 for details of the topics covered in the training). This protocol was a more streamlined version of the one reported in the Singh et al. (2019) study. The mothers were encouraged to engage in formal meditation for at least $20 \mathrm{~min}$ a day.

MB Condition In this condition, the mothers were taught only the mindfulness and related meditation practices from the 3day stepped care version of the MBPBS protocol (see Table 2). The mothers were encouraged to engage in formal meditation for at least $20 \mathrm{~min}$ a day.

PBS Condition In this condition, only the positive behavior support training from the 3-day stepped care version of the MBPBS protocol was used (see Table 2). Parents learned the PBS principles by working through case examples, and by developing PBS plans for their own children. The PBS principles used in MBPBS are similar to the standard protocols (e.g., Bambara and Knoster 2009), but with a few streamlining adaptations. The key adaptations included reducing the assessments

Table 1 Characteristics of the mothers and their children with ASD in the three experimental conditions

\begin{tabular}{|c|c|c|c|c|c|c|}
\hline & \multicolumn{2}{|l|}{ MBPBS } & \multicolumn{2}{|l|}{ MB } & \multicolumn{2}{|l|}{ PBS } \\
\hline & Mothers & Children & Mothers & Children & Mothers & Children \\
\hline Number of participants & 65 & 65 & 65 & 65 & 65 & 65 \\
\hline Mean age/years (SD) & $40.03(4.08)$ & $12.17(1.62)$ & $36.74(4.63)$ & $11.80(1.65)$ & $38.09(4.83)$ & $11.95(1.68)$ \\
\hline Age range (years) & $33-49$ & $9-14$ & $29-46$ & $9-14$ & $28-48$ & $9-14$ \\
\hline Sex: females & $65(100 \%)$ & 15 & $65(100 \%)$ & 18 & $65(100 \%)$ & 17 \\
\hline \multicolumn{7}{|l|}{ Level of functioning } \\
\hline Average & na & 3 & na & 5 & na & 4 \\
\hline Borderline & na & 16 & na & 18 & na & 19 \\
\hline Mild & na & 46 & na & 42 & na & 42 \\
\hline \multicolumn{7}{|l|}{ ASD level of support } \\
\hline Level 1 & na & 15 & na & 12 & na & 17 \\
\hline Level 2 & na & 50 & na & 53 & na & 48 \\
\hline Number of children on psychotropic medications & na & 29 & na & 26 & na & 27 \\
\hline Number of children with diagnosed mental illness & na & 35 & na & 32 & na & 33 \\
\hline Number of children with behavior plans for aggressive behavior & na & 65 & na & 65 & na & 65 \\
\hline
\end{tabular}

$M B P B S$ Mindfulness-Based Positive Behavior Support, $M B$ mindfulness-based practices, $P B S$ positive behavior support, $A S D$ autism spectrum disorder 
Table 2 Topics covered in the 3day stepped care model of the MBPBS Program
Mindfulness training

PBS training
Samatha, Kinhin, and Open Monitoring meditations

Five hindrances - sensory desire, ill will, sloth and torpor, restlessness and remorse, and doubt

Four immeasurables: lovingkindness, compassion, empathetic joy, equanimity (equipoise)

The three poisons - attachment, anger, and ignorance (doubt)

Beginner's mind

Informal mindfulness practices

Practicing ethical precepts - refrain from (a) harming living creatures, (b) taking that which is not given, and (c) incorrect or false speech

Daily logs and journaling

Guiding principles of positive behavior support (PBS)

Goals for parent, caregiver, or teacher and child

Gathering PBS plan-specific information

Functional assessment tools

Developing specific hypotheses

Designing PBS plans

Function-based modifications

Teaching alternative skills

Changing the consequences

Providing long-term supports to enhance quality of life

Questions for designing PBS plans

Implementing the PBS plans and data collection procedures to just two instruments (i.e., a 10-item Behavioral Assessment Interview Questions and the Questions About Behavioral Function rating scale), teaching the parents to develop and implement the PBS plans without behavior analyst input, focusing the PBS plans on short-term achievable goals, and restricting the length of the PBS plans to no more than 2 pages.

\section{Follow-up Condition}

Follow-up data on outcome variables were collected for 3 years following the termination of 30 weeks of intervention of each experimental condition.

\section{Training}

Across the three experimental conditions, the training was provided in small groups at locations close to the participants, or via telemedicine, and included 5 to 13 participants per group. The training was presented in an informal manner and was very interactive with the mothers participating in asking and answering questions, and actively developing plans for implementation and data collection.

\section{Trainer}

The mindfulness trainer had a lifelong discipline of meditation practices and well over 35 years of experience as a meditation teacher. In addition, the trainer was a trained behavioral analyst, certified at the BCBA-D level, and over 45 years of experience in developing and implementing individualized behavior intervention plans for a wide range of behaviors.

\section{Fidelity of Training}

Training provided in all three experimental conditions was videotaped for $15 \mathrm{~min}$ each hour per day during random segments of the training. Two independent raters, trained in both mindfulness and PBS, rated 24 videotaped segments for fidelity assessment across the three experimental conditions. Two aspects of fidelity were assessed: structural fidelity (i.e., what is being taught) and process fidelity (i.e., how the contents are being taught) (Feagans Gould et al. 2016). Both aspects of fidelity were assessed at $100 \%$ for all three experimental conditions.

\section{Measures}

\section{Training Attendance}

Training staff kept attendance records of the mothers' attendance during the 3-day training for each of the three experimental conditions. 


\section{Meditation Practice}

The mothers were required to keep a daily log of the number of minutes they meditated throughout the study.

\section{Perceived Stress}

The Perceived Stress Scale (PSS-10; Cohen et al. 1983) is a 10 -item rating scale that provides a subjective evaluation of lack of control, unpredictability, and overload in a person's daily life (Cohen and Williamson 1988). The items are rated on a 5-point Likert scale that ranges from 0 (never) to 4 (very often), with higher total score indicating greater stress. PSS-10 has adequate psychometric properties, with Cronbach's alpha of 0.78 (Cohen and Williamson 1988) and 0.80 for the present study. The mothers completed PSS-10 six times: in the first week (week 1) and last week (week 10) of the control condition; in the last week of implementation of all experimental conditions (Week 40); and once a year for 3 years during follow-up.

\section{Aggressive Behavior}

Aggressive behavior was defined as hitting, biting, scratching, punching, kicking, or slapping directed at anyone in the home or community settings. The children exhibited one or more of these behaviors.

\section{Disruptive Behavior}

Disruptive behavior was defined as acts that "negatively affected the family's social interactions, including shoving, inordinate or inappropriate demands for time and attention, creating excessive noise, offensive verbal comments, performing distracting repetitive acts during social interactions, or other idiosyncratic behaviors" (Singh et al. 2014, p. 648) as identified by each mother.

\section{Compliance with Mother's Requests}

Compliance was defined as "responding to mother's requests in a socially appropriate manner within an acceptable timeframe as determined by each mother" (Singh et al. 2014, p. 648).

\section{Data Collection}

Data were collected by the mothers using an app that enabled real-time recording of multiple events. The mothers collected event data when their children were at home or in community settings and in their presence (i.e., only when the mothers were in line of sight of their children). On average, data collection occurred for 4 and $6 \mathrm{~h}$ during the week and 7 to $9 \mathrm{~h}$ during weekends.

\section{Interobserver Agreement}

Interobserver agreement refers to the reliability of data collected simultaneously but independently by two observers (Ledford and Gast 2018). The mothers in this study were the primary observers (i.e., data collectors), and another family member (secondary observer; typically, the fathers) independently collected data for an average of $2 \mathrm{~h}$ per week, during evenings and weekends. For this study, interobserver agreement was defined as both observers recording the same event (i.e., aggressive behavior, disruptive behavior, compliance with mother's requests), within the same time period of observation. Interobserver agreement was calculated by dividing the total number of agreements on events observed by both observers by the total number of events recorded by the primary observer and multiplying by 100 to obtain percentage agreement. The overall interobserver agreements were as follows: MBPBS condition-87\% (range $=81-$ 92\%), MB condition-89\% (range $=79-94 \%$ ), and PBS condition $-88 \%$ (range $=83-95 \%)$.

\section{Data Analyses}

Training attendance and average daily meditation time per week for each group during intervention and follow-up were computed and presented as descriptive statistics. Mothers' perceived stress was analyzed using two-level ANCOVA with three groups (MBPBS, MB, and PBS) as between-subject and six time-points as within-subject factors, and children's age as covariate. Aggressive and disruptive behavior and compliance outcome variables were estimated as mean daily rates per group at each assessment time-point and were analyzed using multiple linear regression (MLR). Binary variables were created for MBPBS, $\mathrm{MB}$, and PBS predictors that were entered in stepwise fashion and followed by meditation practice while controlling for the overall effect of time entered in the first block. Stepwise approach enabled us to estimate which intervention type was the strongest predictor of behavioral outcomes. The strongest predictor was extracted first and the second strongest predictor was extracted after controlling for the effect of the previously extracted predictor until no significant predictors were identified. To estimate a unique effect of meditation practice it was entered into MLR after controlling for time and type of intervention. Statistical significance was determined using conventional criteria of $p<0.05$ throughout and effect sizes were calculated. 
Table 3 Mean daily meditation time per week (in minutes) in MBPBS, $\mathrm{MB}$, and PBS conditions

\begin{tabular}{llll}
\hline Time/group & MBPBS & MB & PBS \\
\hline Control & 0.00 & 0.00 & 0.00 \\
Intervention & 20.17 & 20.07 & 0.00 \\
Year 1 & 25.00 & 22.00 & 0.00 \\
Year 2 & 23.00 & 22.00 & 0.00 \\
Year 3 & 24.00 & 24.00 & 0.00 \\
\hline
\end{tabular}

\section{Results}

Mothers' attendance at the training was $100 \%$. There was 0 min of meditation across the three groups of mothers during the control condition. The mean daily meditation time during intervention and follow-up increased from $20 \mathrm{~min}$ during intervention to $24 \mathrm{~min}$ by the end of the third year of follow-up in both MBPBS and $\mathrm{MB}$ groups but remained at $0 \mathrm{~min}$ for the PBS group. The mean meditation time for each group of mothers during control, intervention, and follow-up is presented in Table 3.

Figure 2 presents the mean perceived stress scores for mothers during intervention and 3 years of follow-up. The largest progressive decline in perceived stress was evident in the MBPBS group followed by the MB group, with the least decline in the PBS group. ANCOVA indicated a significant effect of a group (MBPBS, MB, and PBS), with a large effect size $\left(F_{2,170}=76.64, p<0.001, \eta^{2}=0.47\right)$. There was a significant interaction between time and group $\left(F_{10,170}=196.73\right.$, $p<0.001)$, with an even larger effect size $\left(\eta^{2}=0.69\right)$, indicating that changes in the mothers' perceived stress over time depended largely on type of intervention. The mean perceived stress scores of the three groups were not significantly different at the baseline (control) condition. However, post hoc tests showed that mothers in the MBPBS group were significantly less affected by perceived stress $(M=25.47 ; 95 \%$ CI [24.81, 26.13]) compared with both the MB group $(M=28.93 ; 95 \% \mathrm{CI}$ [28.27, 29.59]) and the PBS group $(M=31.35$; 95\% CI [30.69, 32.01]), which showed significantly higher stress scores among all three groups. The overall significant effect of time was also observed $\left(F_{5,170}=12.99, p<0.001, \eta^{2}=0.07\right)$, with a modest effect size. Furthermore, there was a significant inverse effect of children's age on mother's stress $\left(F_{1,170}=4.95, p=0.027, \eta^{2}=\right.$ 0.03 ) suggesting that younger children cause significantly more stress to their mothers.

Figure 3 shows children's aggressive behavior computed as the mean number of daily events during control, intervention, and follow-up conditions for each group. Although a dramatic decline of aggression was observed in all three groups at the 3year follow-up condition compared with the control condition, complete extinction of aggressive behavior was evident only in the MBPBS group. Also, a greater reduction in aggressive behavior was noted in the MB condition compared with the PBS group. MLR analysis with aggressive events as the outcome variable revealed an overall large effect of time, reflecting shared effectiveness of all three interventions, and explained $28 \%$ of reduction in the outcome $\left(R^{2}=0.28 ; \beta=-\right.$ $0.53 ; p<0.001)$. After controlling for the overall time effect, MBPBS explained an additional $5 \%$ of reduction in aggression $\left(R^{2}=0.05 ; \beta=-0.23 ; p=0.002\right)$. When the effects of time and MBPBS were accounted for, both MB and PBS were not significant predictors, but meditation time explained another $29 \%$ of reduction in the children's aggressive behavior, which is a large effect size $\left(R^{2}=0.29 ; \beta=-0.88 ; p<0.001\right)$.

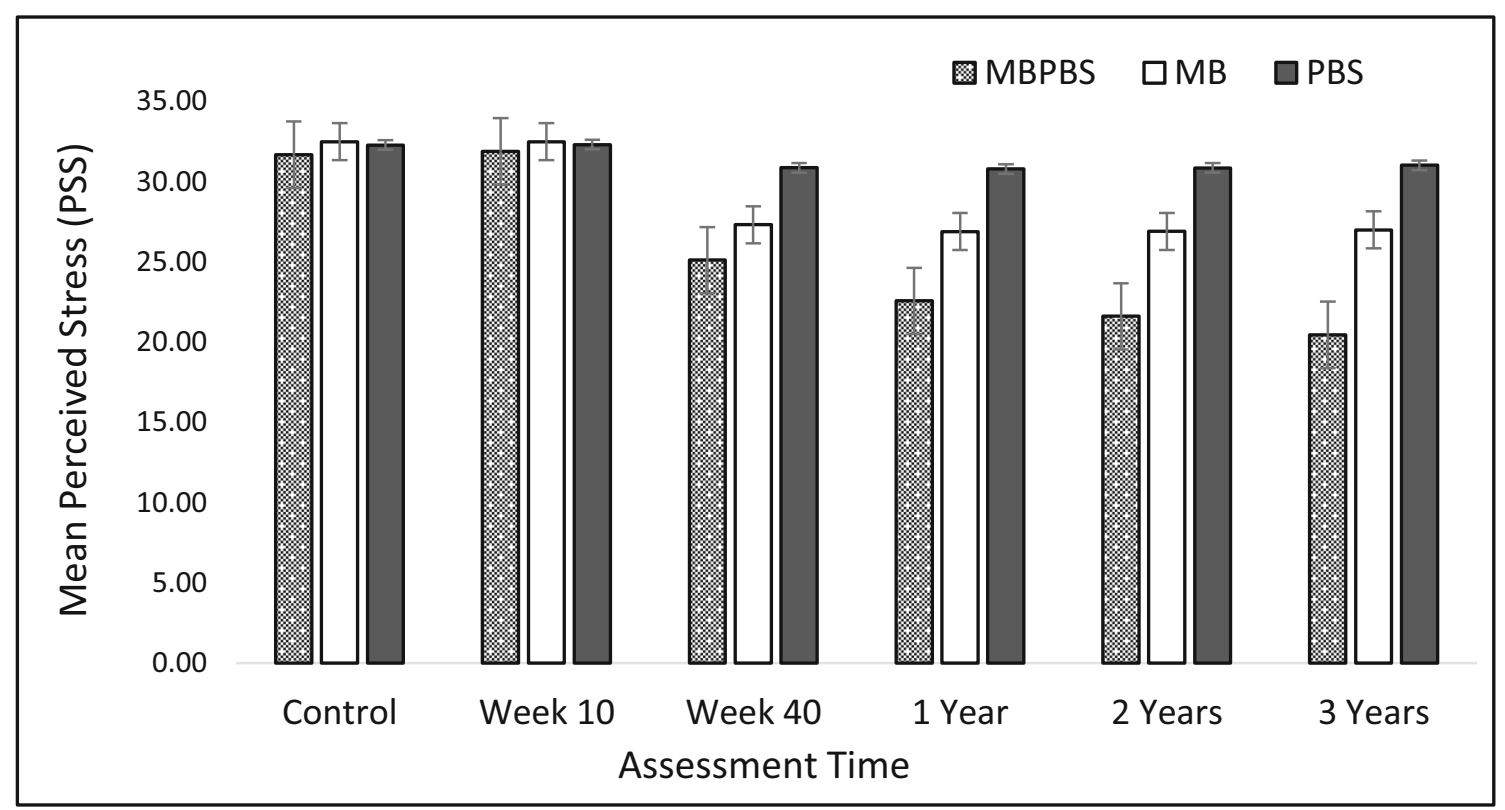

Fig. 2 Mothers' perceived stress mean scores during control, week 10, and week 40 of the intervention and 3-year follow-up. Error bars indicate standard errors of the mean 


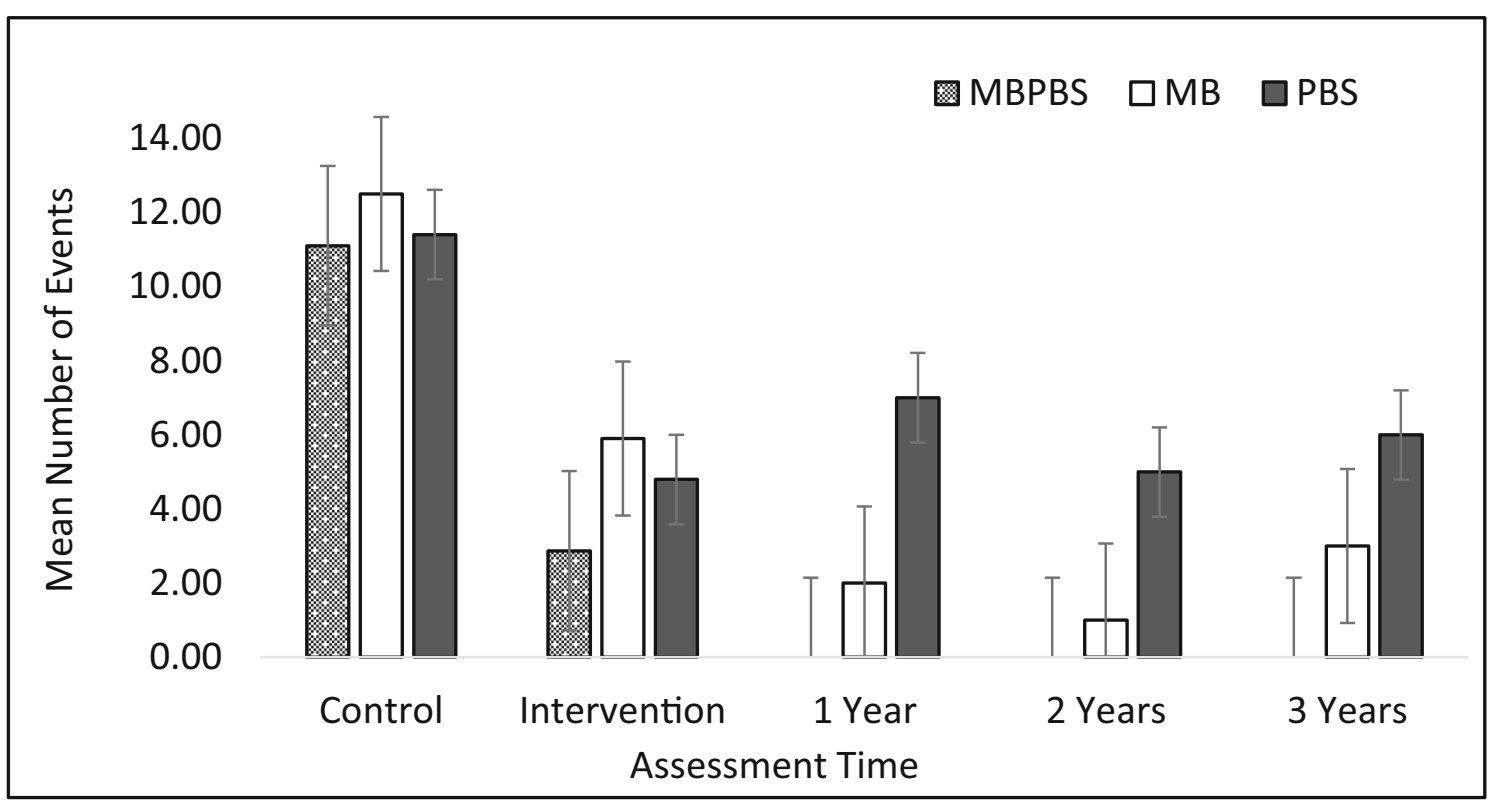

Fig. 3 Mean children's daily aggressive events during control, intervention, and 3-year follow-up. Error bars indicate standard errors of the mean

Children's daily counts of disruptive behavior averaged over control, intervention, and follow-up are presented in Fig. 4, showing strong reduction of disruptive behavior in all three groups at both intervention and follow-up conditions, compared with the control condition. The most profound progressive reduction of disruptive behavior was observed in the MBPBS group followed by the MB group. Even though there was a smaller but still substantial reduction of disruptive behavior observed during intervention in the PBS group, a subsequent reduction was not evident during the 3-year followup. Similar to aggression, MLR revealed the overall large time effect observed in all groups that accounted for $35 \%$ of reduction in disruptive behavior $\left(R^{2}=0.35 ; \beta=-0.59\right.$; $p<0.001)$. MBPBS explained a further $4 \%$ reduction in the outcome $\left(R^{2}=0.04 ; \beta=-0.20 ; p=0.004\right)$ and, by considering this effect, no significant contribution was evident for MB and PBS. When effects of time and type of intervention were controlled for, a large effect of meditation practice was evident explaining $24 \%$ of disruptive behavior decrease $\left(R^{2}=0.24\right.$; $\beta=-0.79 ; p<0.001)$.

Children's daily percentage of compliance averaged for control, intervention, and follow-up conditions is presented in Fig. 5. The greatest increase in compliance, reaching over $80 \%$, was apparent in the MBPBS group and remained stable

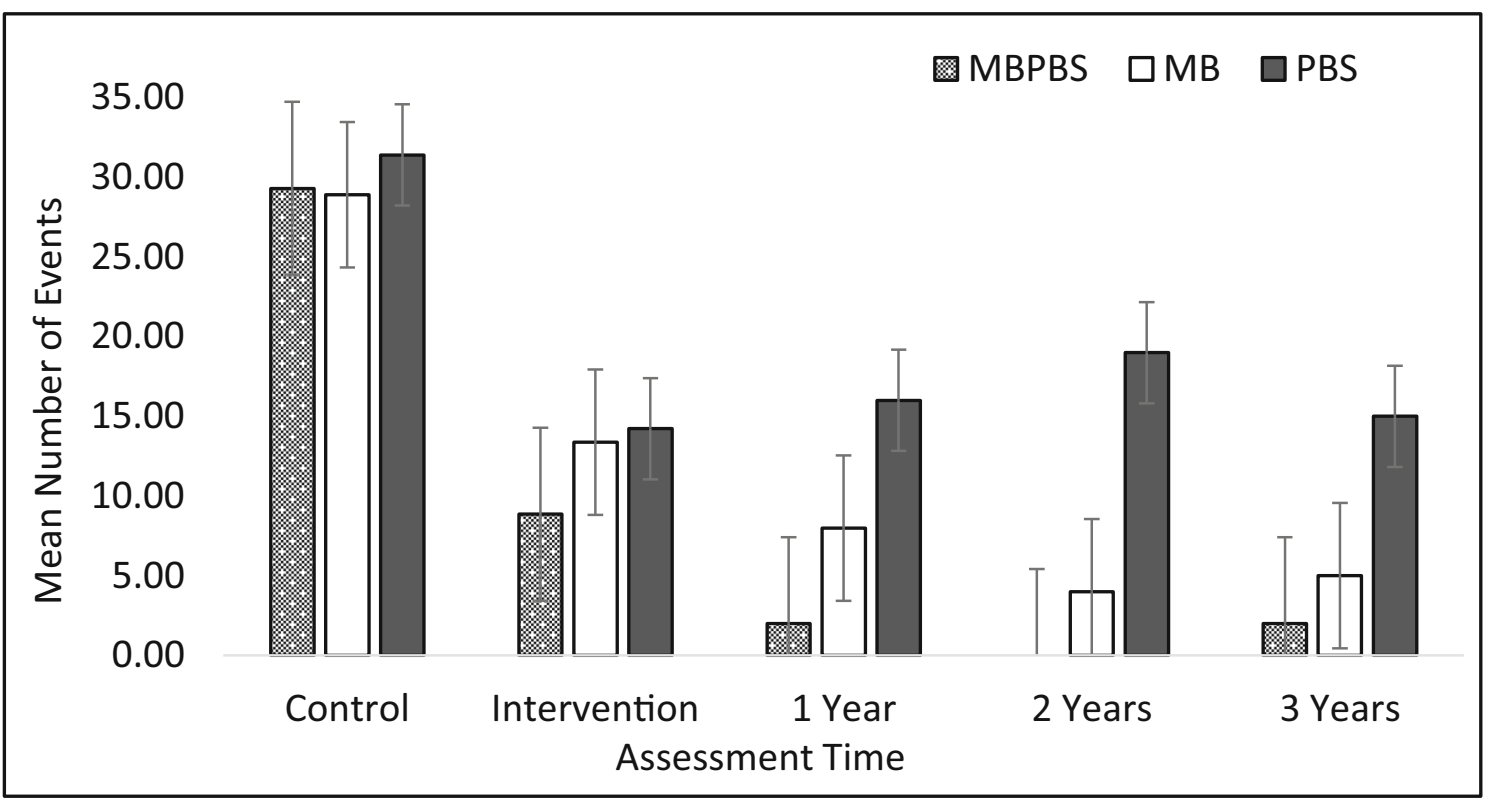

Fig. 4 Mean children's daily disruptive behavior during control, intervention, and 3-year follow-up. Error bars indicate standard errors of the mean 


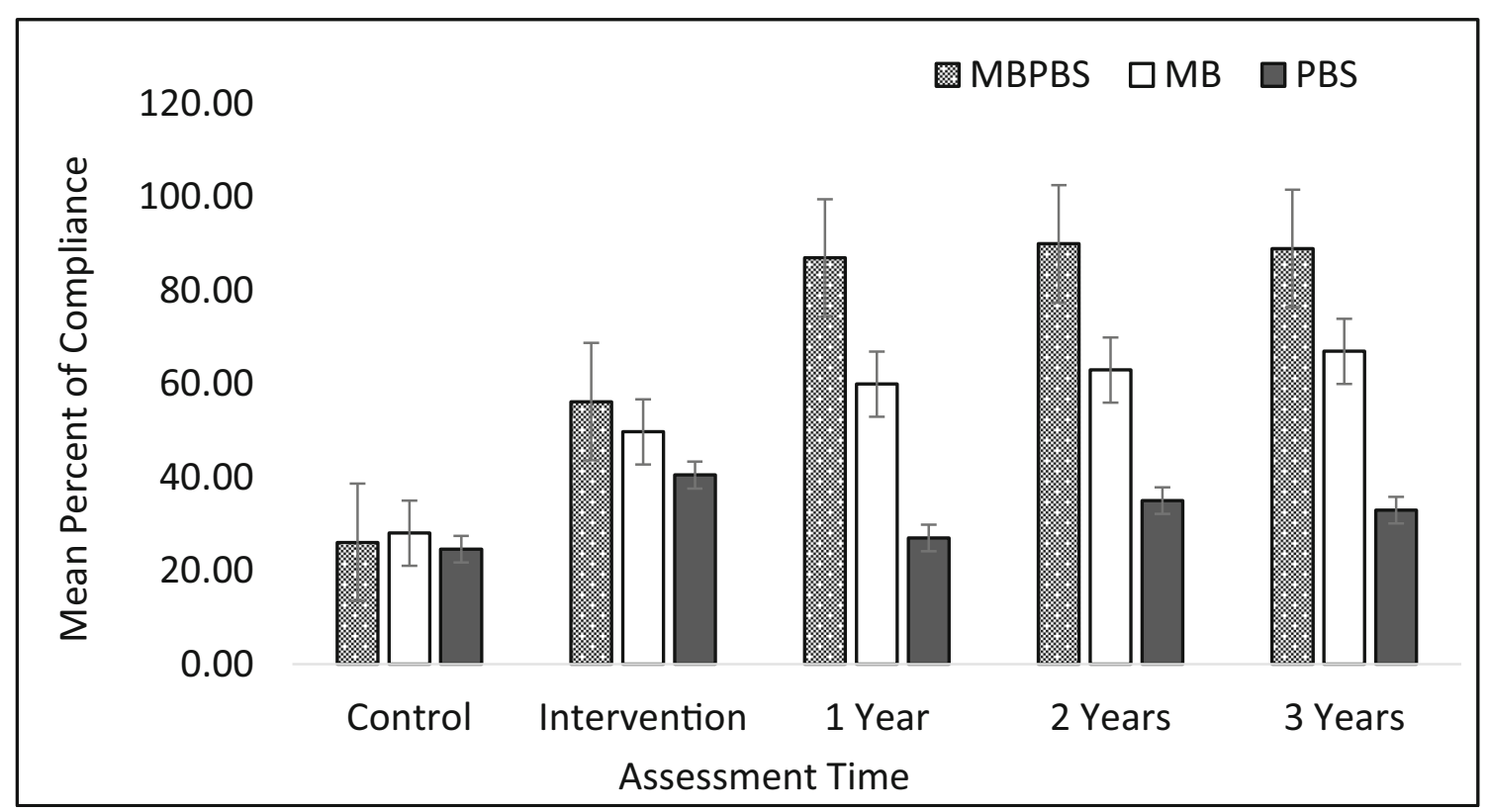

Fig. 5 Mean children's daily percentage compliance during control, intervention, and 3-year follow-up. Error bars indicate standard errors of the mean

during the 3-year follow-up. Children's compliance reached $60 \%$ and remained relatively stable during the 3-year followup period in the MB group. There was an increase of up to $40 \%$ during intervention in the PBS group and then a slight decrease during the follow-up condition. MLR indicated that the overall large effect of time on weekly percentage of compliance accounted for $27 \%$ of the increase $\left(R^{2}=0.27 ; \beta=\right.$ $0.52 ; p<0.001)$, with an additional moderate effect of $8 \%$ explained uniquely by $\operatorname{MBPBS}\left(R^{2}=0.08 ; \beta=0.28\right.$; $p<0.001)$. When effects of time and MBPBS were accounted for, MB made a significant contribution of an additional $5 \%$ $\left(R^{2}=0.05 ; \beta=0.26 ; p=0.002\right)$. Finally, a large effect of meditation practice was shown after controlling for time and types of interventions $\left(R^{2}=0.27 ; \beta=0.85 ; \mathrm{p}<0.001\right)$.

\section{Discussion}

There is substantial research that attests to the effectiveness of parent training programs in the treatment of disruptive behavior in children with ASD (Lecavalier et al. 2017; Postorino et al. 2017). In addition, parent management training has been shown to be significantly effective in the "real world" as well as in randomized controlled trials (Michelson et al. 2013). These programs are designed to enhance the role of parents as the behavior change agents in the lives of their children. While effective, some parents still evidence maternal burnout syndrome because they find parenting to be stressful, and exhibit depression and anxiety (Lebert-Charron et al. 2018). A suggestion in the parenting literature has been that behavioral parent training programs do not focus on the mother's stress but concentrate mainly on teaching them how to effectively manage their child's behavior (Yu et al. 2019). Furthermore, behavioral programs focusing on the child's challenging behaviors mandate customized programs for each behavior problem because of differences in motivational functions across behaviors, thus increasing the intervention burden on the mothers. Perhaps, we can reduce the mothers' stress and burnout by offering alternative approaches, such as mindful parenting, that have a more general effect not only on their children but also on themselves.

Mindful parenting is the application of mindful attention and awareness to parent-child interactions in everyday family life (Kabat-Zinn and Kabat-Zinn 2014). In mindful parenting programs, parents learn how to mindfully take care of themselves and their children (Bögels and Restifo 2014; Hwang and Kearney 2015). A closely related approach is to combine mindful parenting with mindfulness training for the children and adolescents (e.g., the MYmind program; de Bruin et al. 2015). This may prove particularly advantageous for the children and adolescents because they learn self-care without parental intervention. However, some children may not be ready for this type of training or are so challenged in life that learning a new skill may be too stressful or even beyond their cognitive or emotional abilities. If the child has specific diseases or disorders (e.g., IDD, ASD, ADHD, conduct disorder), a mindful parenting program can be customized to include training in adjunctive interventions (e.g., behavioral interventions, cognitive behavioral procedures, psychoeducational programs) that parents can use to reduce suffering and improve the quality of life of their children. 
MBPBS is an example of a mindfulness-based program that has an adjunctive component, which parents can use to directly influence the nature of their children's behavioral challenges. Extant research indicates that parents, professional caregivers, and teachers can effectively use MBPBS for selfcare, and to produce small mind-shifts in individuals with IDD and ASD such that they can produce large gains in the quality of their lives (Singh et al. 2019). Training in MBPBS produces positive changes in parents, professional caregivers, and teachers on a large number of variables, including stress, burnout, compassion satisfaction, secondary traumatic stress, and job turnover, with collateral positive changes in individuals in their care, including physical and verbal aggression, disruptive behavior, self-injury, social behavior, responsiveness to requests, staff injuries, peer injuries, use of physical restraints, need for emergency medication, and 1-to-1 staffing for behavior problems (e.g., Singh et al. 2013, 2014, 2015, 2016a, 2016b, 2020b).

In a recent study, Singh et al. (2020b) investigated the effects of the PBS component alone when compared with the MBPBS program. The results showed that while both the PBS component alone and the MBPBS programs were effective, MBPBS outperformed PBS on all caregiver, client, and agency variables. This finding aligns well with those reported by Ferraioli and Harris (2013) who demonstrated the superiority of mindful parent training over skills-based parenting. The present study extends these findings by investigating the individual effects of the two components of MBPBS (i.e., MB, PBS) when compared with their combined effects. Furthermore, the participants in the earlier study were professional caregivers of adults who functioned at mild and moderate levels of IDD while, in the present study, the participants were mothers of children with ASD who required level 1 and level 2 support as defined in DSM-5 (American Psychiatric Association 2013). In addition, the present study provides follow-up data over a 3-year period in order to assess maintenance effects produced by MB, PBS, and MBPBS in mothers and their children.

The overall finding of the present study was that the three interventions had differential effects on mother and child outcome variables. Supporting our first hypothesis, the mothers' stress levels decreased the most in the MBPBS group, followed by the MB group, with negligible change in the PBS group. These findings make sense even at the intuitive level given that the PBS approach does not directly target parental stress as a variable to be manipulated. Parental stress may lessen once the level of the children' challenging behavior is reduced, but the stress correlated with the children's challenging behavior to begin with, as well as the labor-intensive nature of implementing PBS plans, was probably too large to overcome in this group. Supporting our second hypothesis, the children's aggressive behavior decreased the most in the MBPBS condition, with the effects being greater than the individual effects of MB and PBS combined. This suggests that the synergistic effects when MB and PBS are presented together as a seamless process produce enhanced outcomes when compared with the effects of MB and PBS alone. Furthermore, greater decreases in aggressive behavior were correlated with increasing meditation practice by the mothers. The data for disruptive behavior mirrored those of the children's aggressive behavior in all aspects. Compliance, or the children's responsiveness to mother's requests, increased over time, with significantly larger increases in the MBPBS and MB groups. In this longitudinal study, we were also able to demonstrate that even after excluding effects of time and type of intervention (MB or MBPBS), meditation practice was very powerful in reducing aggressive and disruptive behaviors of the children, and in enhancing their compliance with mother's requests. Although there are evidence-based behavioral antecedent strategies (e.g., high-probability command sequences, effective instruction delivery, and errorless compliance training) that can be used to promote compliance with requests in children and adolescents (Radley and Dart 2016), the present study suggests that parental mindfulness can achieve the same ends without direct instruction.

The present study adds to the research literature on mindful parenting based on MBPBS, a program that has been shown to benefit not only parents but also professional caregivers and teachers. When parents engage in the MBPBS program, there is evidence of direct effects on their own behavior, which then results in positive spillover effects on their children. This spillover effect has been reported in other mindful parenting programs as well (e.g., Meppelink et al. 2016). There are compelling empirical evidence and theoretical explanations of behavioral spillover effects in the psychological research literature (Dolan and Galizzi 2015) to suggest that this may be the case with the MBPBS program. The spillover or cascading effects of parent mindfulness have positive effects on the children's behaviors in terms of decreasing challenging behaviors and increasing their responsiveness to parental requests. In a previous study (Singh et al. 2020b), and in the present study, the data indicated that MBPBS as a package of meditations and specific instructions in PBS produces the largest spillover effects on the children followed by the MB component, with the PBS component alone being less effective than either the MBPBS program or the MB component alone. We can speculate that it is the meditation component of the MBPBS program, which may be the key ingredient that makes both the MBPBS program and the MB component alone more effective than the PBS component alone. However, this needs to be empirically verified in future research. Furthermore, better understanding of which parent characteristics mediate enhanced outcomes is needed to ensure the MBPBS program specifically targets these characteristics to increase the effectiveness of the program (see Shalev et al. 2020). 


\section{Strengths, Limitations, and Future Research Directions}

This study adds to the research base on the efficacy and effectiveness of MBPBS in mindful parenting. It provides further data on component analysis of the multicomponent program, showing that the two key components are individually effective but when combined the effects of the program are greater than the sum of its parts. The study also provides postintervention follow-up data for 3 years showing the lasting effects of initial training in MBPBS. This suggests that once mindful parenting was well-established in the mothers, it put mother-child interactions on a positive trajectory that continued well after the initial training.

This study is not without limitations. The participants were mothers and included no fathers, thus restricting generalizability across parents. There has been a paucity of fathers in mindful parenting research generally, but more effort needs to be expanded to overcome this issue. Parental distress was assessed using only one well-validated measure of perceived stress (PSS-10) and adding measures on anxiety and depression would permit evaluation of the effects of MBPBS and its components on these affective facets of distress. In addition, measuring distress symptoms in children would reveal potential spillover effects of the mothers' training and practice of mindfulness on affective distress symptoms in their children.

There has been much discussion regarding the emergence of a second generation of mindfulness-based programs that are more closely aligned with the Buddhist roots of mindfulness (Van Gordon and Shonin 2020). One of the consequences of this movement has been the need to add more components to existing programs or developing new programs that are more closely aligned with Buddhist practices. This expansion needs to be juxtaposed against the time and effort needed by practitioners (e.g., parents) to learn, metabolize, and use these practices in their already rather full daily lives. We need to learn more about parental needs, motivations, and their characteristics that mediate their parenting practices to customize mindful parenting programs (Emerson et al. 2020). The stepped care model used in developing the MBPBS program is one way of matching the content of mindfulness programs to the needs and abilities of parents.

Acknowledgements A substantial portion of the data included in this paper were first presented at the International Conference on Mindfulness, University of Amsterdam, Amsterdam, The Netherlands, July $10-13,2018$.

\section{Compliance with Ethical Standards}

Conflict of Interest Nirbhay N. Singh is the developer of the MBPBS program. The authors declare no conflict of interest and they do not work for, consult to, and own shares in or receive funding from any company or organization that would benefit from this article.
Ethical Approval All procedures performed in studies involving human participants were in accordance with the ethical standards of the institutional and/or national research committee and with the 1964 Helsinki declaration and its later amendments or comparable ethical standards.

Informed Consent Informed consent was obtained from all individual participants included in the study.

\section{References}

American Psychiatric Association. (2013). Diagnostic and statistical manual of mental disorders (5th ed.). Arlington, VA: author.

Bagner, D. M., \& Eyberg, S. M. (2007). Parent-child interaction therapy for disruptive behavior in children with mental retardation: a randomized controlled trial. Journal of Clinical Child and Adolescent Psychology, 36(3), 418-429.

Bagner, D. M., Pettit, J. W., Lewinsohn, P. M., \& Seeley, J. R. (2010). Effect of maternal depression on child behavior: a sensitive period? Journal of the American Academy of Child \& Adolescent Psychiatry, 49(7), 699-707.

Bagner, D. M., Pettit, J. W., Lewinsohn, P. M., Seeley, J. R., \& Jaccard, J. (2013). Disentangling the temporal relationship between parental depressive symptoms and early child behavior problems: a transactional framework. Journal of Clinical Child and Adolescent Psychology, 42(1), 78-90.

Bambara, L. M., \& Knoster, T. P. (2009). Designing positive behavior support plans (2nd ed.). Washington, DC: American Association on Intellectual and Developmental Disabilities.

Bögels, S., \& Restifo, K. (2014). Mindful parenting: a guide for mental health practitioners. New York, NY: Springer.

Cachia, R. L., Anderson, A., \& Moore, D. W. (2016). Mindfulness, stress and wellbeing in parents of children with autism spectrum disorder: a systematic review. Journal of Child and Family Studies, 25, 1-14.

Cohen, S., Kamarck, T., \& Mermelstein, R. (1983). A global measure of perceived stress. Journal of Health and Social Behavior, 24(4), 385 396.

Cohen, S., \& Williamson, G. (1988). Psychological stress in a probability sample of the United States. In S. Spacapan \& S. Oskamp (Eds.), The social psychology of health: Claremont symposium on applied social psychology (pp. 31-63). Newbury Park, CA: Sage.

de Bruin, E. I., Blom, R., Smit, F. M. A., van Steensel, F. J. A., \& Bögels, S. (2015). MYmind: mindfulness training for youngsters with autism spectrum disorders and their parents. Autism, 19(8), 906-914.

Dolan, P., \& Galizzi, M. M. (2015). Like ripples on a pond: behavioral spillovers and their implications for research and policy. Journal of Economic Psychology, 47, 1-16.

Emerson, L. M., Aktar, E., de Bruin, E., Potharst, E., \& Bögels, S. (2020). Mindful parenting in secondary child mental health: key parenting predictors of treatment effects. Mindfulness. Advance of Print.

Feagans Gould, L., Dariotis, J. K., Greenberg, M. T., \& Mendelson, T. (2016). Assessing fidelity of implementation (FOI) for school-based mindfulness and yoga interventions: a systematic review. Mindfulness, 7(1), 5-33.

Ferraioli, S. J., \& Harris, S. L. (2013). Comparative effects of mindfulness and skills-based parent training programs for parents of children with autism: feasibility and preliminary outcome data. Mindfulness, 4, 89-101.

Fowler, J. H., \& Christakis, N. A. (2010). Cooperative behavior cascades in human social networks. PNAS, 107(12), 5334-5338.

Hastings, R. P., Kovshoff, H., Ward, N. J., degli Espinosa, F., Brown, T., \& Remington, B. (2005). Systems analysis of stress and positive perceptions in mothers and fathers of pre-school children with 
autism. Journal of Autism and Developmental Disorders, 35(5), 635-644.

Hayes, S. A., \& Watson, S. L. (2013). The impact of parenting stress: a meta-analysis of studies comparing the experience of parenting stress in parents of children with and without autism spectrum disorder. Journal of Autism and Developmental Disorders, 43(3), 629642.

Hwang, Y.-S., \& Kearney, P. (2015). A mindfulness intervention for children with autism spectrum disorder: new directions in research and practice. New York, NY: Springer.

Hwang, Y-S., \& Singh, N. N. (2016). Mindfulness. In N. N. Singh (Ed.), Handbook of evidence-based practices in intellectual and developmental disabilities (pp. 311-346). New York, NY: Springer.

Kabat-Zinn, M., \& Kabat-Zinn, J. (2014). Everyday blessings: the inner work of mindful parenting (Rev. ed.). New York, NY: Hachette Books.

Kleve, L., Crimlisk, S., Shoebridge, P., Greenwood, R., Baker, B., \& Mead, B. (2011). Is the Incredible Years programme effective for children with neurodevelopmental disorders and for families with social services involvement in the "real world" of community CAMHS? Clinical Child Psychology and Psychiatry, 16(2), 253264.

Lebert-Charron, A., Dorard, G., Boujut, E., \& Wendland, J. (2018). Maternal burnout syndrome: contextual and psychological factors. Frontiers in Psychology, 9, 885.

Lecavalier, L., Smith, T., Johnson, C., Bearss, K., Swiezy, N., Aman, M. G., et al. (2017). Moderators of parent training for disruptive behaviors in young children with autism spectrum disorder. Journal of Abnormal Child Psychology, 45, 1235-1245.

Ledford, J. R., \& Gast, D. J. (2018). Single case research methodology. New York, NY: Routledge.

McIntyre, L. L. (2008a). Adapting Webster-Stratton's Incredible Years Parent Training for children with developmental delay: findings from a treatment group only study. Journal of Intellectual Disability Research, 52, 1176-1192.

McIntyre, L. L. (2008b). Parent training in young children with developmental disabilities: a randomized controlled trial. American Journal on Mental Retardation, 113, 356-368.

McIntyre, L. L., \& Neece, C. L. (2016). Parent training. In N. N. Singh (Ed.), Handbook of evidence-based practices in intellectual and developmental disabilities (pp. 467-492). New York, NY: Springer.

Meppelink, R., de Bruin, E. I., Wanders-Mulder, F. H., Vennik, C. J., \& Bögels, S. M. (2016). Mindful parenting training in child psychiatric settings: heightened parental mindfulness reduces parents' and children's psychopathology. Mindfulness, 7, 680-689.

Michelson, D., Davenport, C., Dretzke, J., Barlow, J., \& Day, C. (2013). Do evidence-based interventions work when tested in the "real world?" A systematic review and meta-analysis of parent management training for the treatment of child disruptive behavior. Clinical and Child Family Psychology Review, 16(1), 18-34.

Neece, C. L. (2014). Mindfulness-based stress reduction for parents of young children with developmental delays: implications for parental mental health and child behavior problems. Journal of Applied Research on Intellectual Disabilities, 27(2), 174-186.

Plant, K. M., \& Sanders, M. R. (2007). Reducing problem behavior during caregiving in families of preschool-aged children with developmental disabilities. Research in Developmental Disabilities, 28, $362-385$.

Poole, J. (2003). Tackling poor parenting: a public health issue. Journal of Family Health, 13, 49-51.

Postorino, V., Sharp, W. G., McCracken, C. E., Bearss, K., Burrell, T. L., Evans, A. N., \& Scahill, L. (2017). A systematic review and metaanalysis of parent training for disruptive behavior in children with autism spectrum disorder. Clinical Child and Family Psychology Review, 20, 391-402.
Radley, K. C., \& Dart, E. H. (2016). Antecedent strategies to promote children's and adolescent's compliance with adult requests: a review of the literature. Clinical Child and Family Psychology Review, 19, $39-54$.

Roskam, I., Raes, M. E., \& Mikolajczak, M. (2017). Exhausted parents: development and preliminary validation of the parental burnout inventory. Frontiers in Psychology, 8, 163.

Shalev, R. A., Lavine, C., \& Di Martino, A. (2020). A systematic review of the role of parent characteristics in parent-mediated interventions for children with autism spectrum disorder. Journal of Developmental and Physical Disabilities, 32, 1-21.

Singh, N. N., Lancioni, G. E., Chan, J., McPherson, C. L., \& Jackman, M. M. (2020a). Mindfulness-Based Positive Behavior Support. In I. Ivtzan (Ed.), Handbook of mindfulness-based programs: mindfulness interventions from education to health and therapy (pp. 42-52). London, UK: Routledge.

Singh, N. N., Lancioni, G. E., Karazsia, B. T., Chan, J., \& Winton, A. S. W. (2016b). Effectiveness of caregiver training in mindfulnessbased positive behavior support (MBPBS) vs. training-as-usual (TAU): a randomized controlled trial. Frontiers in Psychology, 7, 1549.

Singh, N. N., Lancioni, G. E., Karazsia, B. T., \& Myers, R. E. (2016a). Caregiver training in Mindfulness-Based Positive Behavior Supports (MBPBS): effects on caregivers and adults with intellectual and developmental disabilities. Frontiers in Psychology, 7, 98.

Singh, N. N., Lancioni, G. E., Karazsia, B. T., Myers, R. E., Hwang, Y.S., \& Anālayo, B. (2019). Effects of Mindfulness-Based Positive Behavior Support (MBPBS) training are equally beneficial for mothers and their children with autism spectrum disorder or with intellectual disabilities. Frontiers in Psychology, 10, 385.

Singh, N. N., Lancioni, G. E., Karazsia, B. T., Myers, R. E., Winton, A. S. W., Latham, L. L., \& Nugent, K. (2015). Effects of training staff in MBPBS on the use of physical restraints, staff stress and turnover, staff and peer injuries, and cost effectiveness in developmental disabilities. Mindfulness, 6, 926-937.

Singh, N. N., Lancioni, G. E., Medvedev, O. N., Myers, R. E., Chan, J., McPherson, C. L., Jackman, M. M., \& Kim, E. (2020b). Comparative effectiveness of caregiver training in mindfulnessbased positive behavior support (MBPBS) and positive behavior support (PBS) in a randomized controlled trial. Mindfulness, 11(1), 99-111.

Singh, N. N., Lancioni, G. E., Winton, A. S., Fisher, B. C., Wahler, R. G., McAleavey, K., et al. (2006). Mindful parenting decreases aggression, noncompliance, and self-injury in children with autism. Journal of Emotional and Behavioral Disorders, 14, 169-177.

Singh, N. N., Lancioni, G. E., Winton, A. S. W., Karazsia, B. T., Myers, R. E., Latham, L. L., et al. (2014). Mindfulness-based positive behavior support (MBPBS) for mothers of adolescents with autism spectrum disorder: effects on adolescents' behavior and parental stress. Mindfulness, 5, 646-657.

Singh, N. N., Lancioni, G. E., Winton, A. S. W., Karazsia, B. T., \& Singh, J. (2013). Mindfulness training for teachers changes the behavior of their preschool students. Research in Human Development, 10(3), 211-233.

Singh, N. N., Lancioni, G. E., Winton, A. S. W., Singh, J., Curtis, J. W., Wahler, R. G., \& McAleavey, K. M. (2007). Mindful parenting decreases aggression and increases social behavior in children with profound developmental disabilities. Behavior Modification, 31, $749-771$.

Singh, N. N., Singh, A. N., Lancioni, G. E., Singh, J., Winton, A. S. W., \& Adkins, A. D. (2010). Mindfulness training for parents and their children with ADHD increases the children's compliance. Journal of Child and Family Studies, 19, 157-166.

Stone, L. L., Mares, S. H. W., Otten, R., Engels, R., \& Janssens, J. (2016). The co-development of parenting stress and childhood internalizing 
and externalizing problems. Journal of Psychopathology and Behavioral Assessment, 38(1), 76-86.

ten Hoopen, L. W., de Nijs, P. F., Duvekot, J., Greaves-Lord, K., Hillegers, M. H. J., Brouwer, W. B. F., \& Hakkaart-van Roijen, L. (2020). Children with an autism spectrum disorder and their caregivers: capturing health-related and care-related quality of life. Journal of Autism and Developmental Disorders, 50, 263-277.

Van Gordon, W., \& Shonin, E. (2020). Second-generation mindfulnessbased interventions: toward more authentic mindfulness practice and teaching. Mindfulness, 11(1), 1-4.

Whittingham, K., Sofronoff, K., Sheffield, J., \& Sanders, M. R. (2009). Stepping Stones Triple P: an RCT of a parenting program with parents of a child diagnosed with autism spectrum disorder. Journal of Abnormal Child Psychology, 37, 469-480.
Yorke, I., White, P., Weston, A., Rafla, M., Charman, T., \& Simonoff, E. (2018). The association between emotional and behavioral problems in children with autism spectrum disorder and psychological distress in their parents: a systematic review and meta-analysis. Journal of Autism and Developmental Disorders, 48, 3393-3415.

Yu, Y., McGrew, J. H., \& Boloor, J. (2019). Effects of caregiver-focused programs on psychosocial outcomes in caregivers of individuals with ASD: a meta-analysis. Journal of Autism and Developmental Disorders, 49, 4761-4779.

Publisher's Note Springer Nature remains neutral with regard to jurisdictional claims in published maps and institutional affiliations. 